\title{
An electronic surgical order, undertaking patient education, and obtaining informed consent for regional analgesia before the day of surgery reduce block-related delays
}

This article was published in the following Dove Press journal:

Local and Regional Anesthesia

5 October 2016

Number of times this article has been viewed

\author{
Brandon S Brooks' \\ Joydip Barman² \\ Brent A Ponce ${ }^{3}$ \\ Alisa Sides ${ }^{4}$ \\ Thomas R Vetter' \\ 'Department of Anesthesiology and \\ Perioperative Medicine, ${ }^{2}$ Department \\ of Health Care Organization and \\ Policy, ${ }^{3}$ Division of Orthopaedic \\ Surgery, Department of Surgery, \\ University of Alabama at Birmingham, \\ Birmingham, AL, ${ }^{4}$ Community Health \\ Systems, Franklin, TN, USA
}

\begin{abstract}
Background: Obtaining patient informed consent for a regional analgesia block on the day of surgery can result in surgical case delays. We hypothesized that implementing a preoperative electronic surgical order, undertaking patient education, and obtaining informed consent for a regional block in our preoperative assessment clinic prior to the day of surgery would reduce surgical case delays attributed to our regional anesthesia pain service and increase the percentage of patients for whom our regional anesthesia pain service was requested to provide a block. Methods: A prospective two-group time-series design, with a nonrandomized, pre- and postintervention data collection strategy, was applied. Based upon the surgeons' newly implemented preoperative electronic outpatient orders, patients were identified by our preoperative assessment clinic staff to receive educational materials. The attending anesthesiologist in the preoperative assessment clinic then obtained written informed consent. Block-related delay and utilization data were analyzed with conventional inferential statistics.
\end{abstract}

Results: We observed a $14.8 \%(95 \%$ CI: $9.4 \%, 20.1 \%$; $P<0.001)$ decrease in surgical case delays, attributed to the regional nerve block, in the post- vs pre-intervention group. In addition, there was a $9.9 \%(95 \% \mathrm{CI}: 4.7 \%, 15.1 \%) ; P<0.001)$ increase in the proportion of patients for whom a regional nerve block was ordered by our three high-volume orthopedic surgeons in the post- vs pre-intervention time periods.

Conclusion: When performed before the day of surgery, a surgeon's electronic order, patient education, and informed consent for regional postoperative analgesia can improve patient throughput, thereby reducing block-related operating room delays. The preoperative assessment clinic can serve as a venue to achieve this goal, thereby adding value by decreasing downstream delays on the day of surgery.

Keywords: regional analgesia, peripheral nerve blockade, informed consent, patient education, performance improvement

\section{Introduction}

Regional nerve blockade has become widely applied for orthopedic surgery. Its reported advantages include reduced opioid requirements, earlier ambulation and hospital discharge, enhanced rehabilitation, and less frequent persistent postsurgical pain. ${ }^{1}$

Obtaining informed consent for a regional nerve block involves a detailed discussion of the technique, risks/benefits, and expectations with a patient who may be unaware of its planned use for postoperative analgesia. This can lead to confusion and anxiety
Department of Anesthesiology and Perioperative Medicine, University of Alabama at Birmingham, 619, 19th Street South, JT862, Birmingham, AL 35249-

6810, USA

$\mathrm{Tel}+\mathrm{I} 205934650$ I

Fax + I 2059967272

Email tvetter@uabmc.edu 
in a patient expecting only general anesthesia. ${ }^{2}$ Preoperative multimedia information can reduce the anxiety of surgical patients undergoing regional anesthesia. ${ }^{3}$

Furthermore, the time needed for adequate patient education and consent can result in case delays on the day of surgery. Because they perceived that our regional anesthesia pain service (RAPS) was causing excessive surgical case delays, three high-volume orthopedic surgeons at our institution demonstrated an increasing unwillingness to have a preoperative block performed on their patients for postoperative analgesia. An identified solution was to undertake the patient informed consent process for a regional analgesia block prior to the day of surgery.

No data have been published on the benefits of obtaining patient consent for regional analgesia blockade prior to the day of surgery. The primary aim of this study was to assess the effect of the regional analgesia block consent process occurring prior to the day of surgery. We hypothesized that implementing a preoperative electronic surgical order, in addition to undertaking patient education and obtaining informed consent for a regional analgesia block, prior to the day of surgery in our Preoperative Assessment, Consultation, and Treatment (PACT) Clinic would 1) reduce the surgical case delays attributed to our RAPS and 2) increase the percentage of patients of our three high-volume orthopedic surgeons for whom our RAPS provided a block.

\section{Methods}

This continuous quality improvement study was approved by the University of Alabama at Birmingham (UAB) Institutional Review Board (IRB) (E110311001). This study was granted exempt status by the UAB IRB because the research involved existing data from January 1, 2013, through February 28, 2015, and involved minimal risk to the study participants. A waiver of informed consent documentation was granted by the UAB IRB. This study was an observational (before and after) care redesign study. It was not a prospective clinical trial and hence was not registered.

A two-group time-series design, with a nonrandomized, pre- and post-intervention data collection strategy, was applied in this prospective observational study. ${ }^{4}$ The primary outcome was a regional nerve block-related orthopedic surgical case delay. The secondary outcome was whether an orthopedic surgery patient deemed eligible for a regional nerve block was requested to receive a block by one of the three high-volume orthopedic surgeons. These three high-volume surgeons were collectively performing $\sim 1,700$ cases/year.
In our pre-intervention study group $(\mathrm{N}=388,12$-month period from January 1, 2013, to December 31, 2013), an ad hoc discussion occurred on a case-by-case basis, on the day of surgery, between the attending anesthesiologist assigned to our RAPS and the orthopedic surgeon, to determine whether a patient was a candidate for a nerve block for postoperative analgesia. The patient was then approached and educated about the potential nerve block. Patient written informed consent for this block was then obtained on the day of surgery.

Our new preoperative electronic surgical order, patient education, and consent processes were developed in 2013 and were implemented and piloted in January and February 2014. In our post-intervention study group ( $N=425,12$-month period from March 1, 2014, to February 28, 2015), as part of their electronic preoperative order set completed in their clinic, our orthopedic surgeons requested that their elective surgical patients, who were deemed eligible, receive a singledose or a continuous catheter nerve block for postoperative analgesia. These patients were then identified by our PACT Clinic nursing staff, who initiated the new patient-centered process.

While in our PACT Clinic, to facilitate their informed consent process, all so-identified patients were educated about regional analgesic blockade by watching an Apple $\mathrm{iPad}^{\circledR}$ Inc.'s (Cupertino, CA, USA) based video and receiving an informational brochure. The attending anesthesiologist in the PACT Clinic then answered any remaining patient questions and obtained the patient's written informed consent for the block. The patient was noted on the operating room schedule as having been ordered to receive and consented for a nerve block. On the day of surgery, the attending anesthesiologist assigned to the dedicated RAPS confirmed the patient's continued desire for a nerve block and answered any remaining patient questions before proceeding.

There were no other changes in the RAPS process "between" the pre- and post-intervention study periods, including no change in its attending/resident staffing ratios; no change in the nerve block-related location, allocated space, equipment, or support staff; no earlier morning shift start time by the regional block team; and no standardized earlier arrival and check-in of our surgical patients. The same day surgery nurse's patient intake and the surgeon's 24-hour update and site-marking processes were also unchanged.

We focused on delays in first case on-time starts, as this was an organizational initiative to improve. A surgical case was defined as delayed if it started $>5$ minutes after the posted/scheduled time. One of the six possible causes for all surgical case delays (surgeon, patient, operating room, 
anesthesia, preoperative nursing, or RAPS) was assigned by the circulating surgical nurse and confirmed by the Director of Perioperative Services, in a consistent manner in both the study groups. There was no more specific definition or focus on the assignment of case delay after January 2014. Data were abstracted from our institutional administrative (GE Centricity ${ }^{\mathrm{TM}}$ Business; GE Healthcare Bio-Sciences Corp., Piscataway, NJ, USA) and electronic medical record (PowerInsight $^{\circledR}$; Cerner Corp., Kansas City, MO, USA) sources.

\section{Statistical analysis}

Although this new preoperative regional block electronic order and new patient education/consent program included all of our orthopedic surgeons, for the comparison of the regional anesthesia service consult request rates, only data from the subset of the three targeted high-volume orthopedic surgeons were analyzed.

Continuous variables were reported using mean and standard deviation, or if the data were skewed as a median and interquartile range. Parametric continuous demographic data were compared between groups using a $t$-test with nonequal variances. Nonparametric data were compared using a Mann-Whitney $U$-test. Categorical variables were reported using frequency counts and percentages. Categorical data were compared between groups using a chi-square test or Fisher's exact test when indicated. A $P$-control chart was generated to assess the proportion of block-related delays in the entire study sample.

A logistic regression model was created with the dependent variable being nerve block-related delay (yes/no) to evaluate the effect of being in the post- vs pre-intervention group, adjusting for the covariates of location of the nerve block (upper extremity or lower extremity), the type of nerve block (single dose or continuous perineural catheter), and whether ultrasound guidance (yes/no) was used to perform the nerve block.

Continuous data were assessed for normality with a Shapiro-Wilk test and by examining $Q-Q$ plots, and if nonparametric, they were analyzed as such. No a priori sample size determination and power analysis were performed. For all univariate and logistic regression analyses, a $P$-value of $<0.05$ was considered significant. Statistical analyses were performed using SAS ${ }^{\circledR}$ Version 9.3 (SAS Institute Inc., Cary, NC, USA).

\section{Results}

There were no statistically significant differences in the demographic and clinical characteristics between the enrolled
Table I Demographic and clinical characteristics of study subjects

\begin{tabular}{|c|c|c|c|}
\hline Variable & $\begin{array}{l}\text { Pre-intervention } \\
\text { group }(\mathrm{N}=\mathbf{3 8 8})\end{array}$ & $\begin{array}{l}\text { Post-intervention } \\
\text { group }(\mathrm{N}=425)\end{array}$ & $P$-value \\
\hline Age, mean $\pm S D$ & $55.7 \pm 15.3$ & $57.2 \pm 14.9$ & 0.154 \\
\hline Sex, N (\%) & & & 0.781 \\
\hline Female & 230 (59) & $256(60)$ & \\
\hline Male & I $58(4 \mid)$ & $169(40)$ & \\
\hline Race, N (\%) & & & 0.488 \\
\hline African American & $8 I(2 I)$ & $99(23)$ & \\
\hline Caucasian & $301(77)$ & $315(74)$ & \\
\hline Hispanic & $5(1)$ & $5(1)$ & \\
\hline Other & $\mathrm{I}(<\mathrm{I})$ & $6(2)$ & \\
\hline $\begin{array}{l}\text { Surgical procedures, } \\
N(\%)\end{array}$ & & & 0.775 \\
\hline Upper extremity & $132(34)$ & $149(35)$ & \\
\hline $\begin{array}{l}\text { procedure vs } \\
\text { lower extremity } \\
\text { procedure }\end{array}$ & $256(66)$ & $276(65)$ & \\
\hline $\begin{array}{l}\text { Type of block, } \\
\mathrm{N}(\%)\end{array}$ & & & 0.941 \\
\hline Single dose & $146(38)$ & $161(38)$ & \\
\hline $\begin{array}{l}\text { Continuous } \\
\text { catheter }\end{array}$ & $242(62)$ & $264(62)$ & \\
\hline $\begin{array}{l}\text { Ultrasound } \\
\text { guidance }\end{array}$ & 145 (37) & $127(30)$ & 0.024 \\
\hline
\end{tabular}

pre- and post-intervention study group patients (Table 1). We observed a significant 14.8\% (95\% CI: 9.4\%, 20.1\%; $P<0.001)$ "absolute" decrease in surgical case delays, attributed to the regional nerve block, in the post-intervention group compared to the pre-intervention group. This represented a 56\% "relative" decrease in regional nerve blockrelated surgical case delays. A $P$-control chart demonstrated a major chronological decrease in statistical variation and correction of special cause variation in the regional nerve block-related surgical case delays for the entire study sample (Figure 1). In addition, there was a 9.9\% (95\% CI: 4.7\%, $15.1 \%) ; P<0.001)$ absolute increase in the proportion of patients for whom a regional nerve block was requested by our three high-volume orthopedic surgeons in the post- vs pre-intervention time periods (Table 2). This represented a $15 \%$ relative increase in the proportion of regional block patients.

Our logistic regression analysis indicated that while controlling for the covariates, block-related surgical case delays in the post-intervention group were $64 \%$ less likely compared to our pre-intervention group (adjusted odds ratio $[\mathrm{aOR}]=0.36 ; 95 \% \mathrm{CI}: 0.25,0.52 ; P<0.001)$. Patients who received a single-dose nerve block were $47 \%$ less likely to experience a block-related surgical case delay compared to patients who received a continuous catheter nerve block (aOR $=0.53 ; 95 \% \mathrm{CI}: 0.35,0.80 ; P=0.002)$. The use of ultrasound 


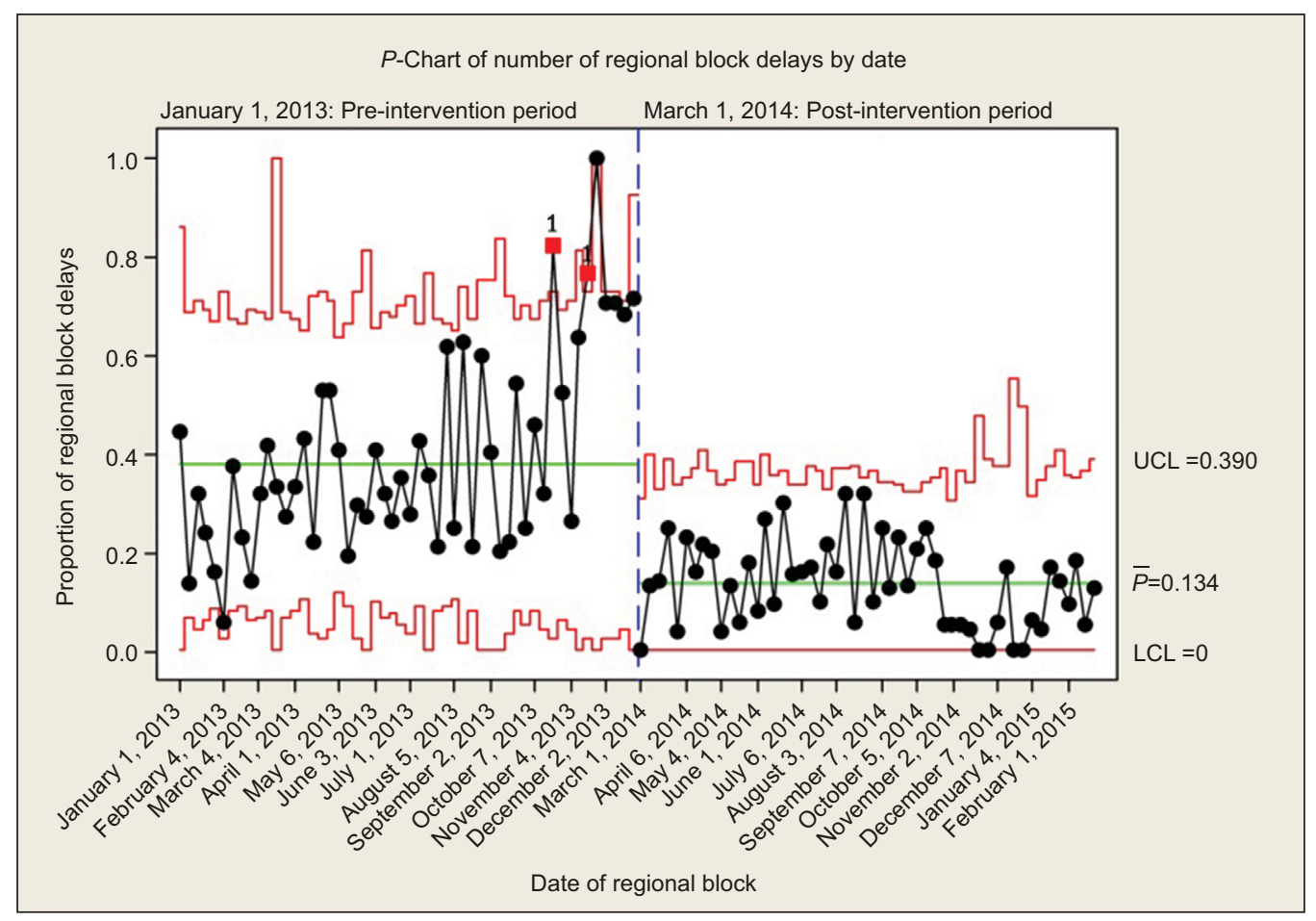

Figure I P-chart of the proportion of all surgical case delays that were attributed to the RAPS performing a preoperative nerve block for all orthopedic surgical cases. Note: $P$-bar $=$ mean proportion.

Abbreviations: LCL, lower control limit; RAPS, regional anesthesia pain service; UCL, upper control limit.

Table 2 Regional nerve block-related primary process outcomes for three high-volume surgeons

\begin{tabular}{llll}
\hline Variable & Post-intervention group & Pre-intervention group & P-value \\
\hline Patients eligible for a block, N & 576 & 550 & $<0.00$ I \\
Eligible patients who received a block, N (\%) & $388(67.4)$ & $425(77.3)$ & $<0.001$ \\
Block patients with surgical case delay, N (\%) & $103(26.6)$ & $50(11.8)$ & \\
\hline
\end{tabular}

Table 3 Univariate and multivariate regression OR for surgical case delay

\begin{tabular}{|c|c|c|c|c|}
\hline Variable & Crude OR $(95 \% \mathrm{Cl})$ & $P$-value & aOR $(95 \% \mathrm{Cl})$ & $P$-value \\
\hline Post-intervention group vs pre-intervention group & $0.37(0.26-0.54)$ & $<0.001$ & $0.36(0.25-0.52)$ & $<0.001$ \\
\hline Single-dose block vs continuous catheter & $0.52(0.35-0.77)$ & 0.001 & $0.53(0.35-0.80)$ & 0.002 \\
\hline Ultrasound guidance (yes vs no) & $0.80(0.54-1.17)$ & 0.240 & $0.83(0.56-1.25)$ & 0.371 \\
\hline Location of nerve block (lower vs upper extremity) & $1.09(0.75-1.58)$ & 0.652 & $1.05(0.71-1.54)$ & 0.806 \\
\hline
\end{tabular}

Abbreviations: aOR, adjusted odds ratio; OR, odds ratio.

guidance $(\mathrm{aOR}=0.83 ; 95 \% \mathrm{CI}: 0.6,1.2 ; P=0.38)$ and the location (upper extremity vs lower extremity) of the nerve block (aOR $=1.0 ; 95 \%$ CI: 0.7, 1.5; $P=0.806$ ) were not associated with a significant decrease in block-related surgical case delays (Table 3).

\section{Discussion}

The potential benefits of regional nerve blockade may be unrealized by surgeons concerned with surgical delays due to block performance. A relative decrease of $>50 \%$ in regional nerve block-related surgical case delays was observed in our post-intervention group compared to our pre-intervention group. In addition, there was a $15 \%$ increase in the proportion of patients for whom a regional block was requested by our three high-volume surgeons in post- vs pre-intervention periods. These results support that the combination of 1) placing a preoperative electronic surgical order and 2) undertaking patient education and obtaining informed consent for a regional analgesia block prior to the day of surgery, reduces surgical case delays attributed to a RAPS, with a concomitant increase in consults requested by orthopedic surgeons.

The triple aim of health care, as espoused by Berwick et $\mathrm{al}^{5}$ and the Institute for Healthcare Improvement, includes ${ }^{6}$ 
1) improving the individual experience of care, 2) improving the health of populations, and 3) reducing per capita costs of care. Achieving these three aims will likely become more important with the advent of value-based perioperative care and expanded pay-for-performance metrics and reimbursement. ${ }^{7,8}$

Patient-centered care and shared decision making ${ }^{9,10}$ are receiving greater attention in the perioperative setting. ${ }^{11,12}$ One practical example is increasing the patient-centered nature of informed consent for procedures. However, a recent study of the quality of informed consent found that prior to a major surgery, $13 \%$ of patients could not recall the procedure to be performed, its indications, risks, or alternatives. ${ }^{13}$ Furthermore, $33 \%$ of patients reported that the decision to proceed did not address their preferences, values, or goals. ${ }^{13}$ In contrast, undertaking the regional block consent process before the day of surgery may be better, as it gives patients more time to reflect and to ask questions, as well as to consider their analgesic options, vs if consented on the day of surgery. Moving this patient consent process to a preoperative assessment clinic is consistent with the triple aim of health care of improving the individual experience of care.

A practical, easy-to-remember three-step shared decisionmaking model has been proposed that can be translated to regional and neuraxial analgesia. ${ }^{14}$ This model has three steps: 1) introducing choice, 2) describing options, often by integrating the use of patient decision support tools, and 3) helping patients explore their preferences and make decisions. This shared decision-making model relies upon supporting a process of deliberation and understanding that decisions be predicated on "what matters most" to the patient. ${ }^{14}$ The present study involved creating and implementing preoperative multimedia patient educational materials as decision support tools. This type of information is easily delivered and can benefit many patients. ${ }^{3}$

Serious neurological complications, including permanent neurological deficits, which are directly related to regional anesthesia/analgesia, continue to be extremely rare. ${ }^{15}$ This is partly attributable to the growing use of ultrasound guidance for regional anesthesia/analgesia over the past 20 years, with an attendant greater number of anesthesiologists trained to use ultrasound guidance. ${ }^{16-18}$ Peripheral nerve blocks are also associated with one or a combination of reduced postoperative pain, reduced opioid consumption, or increased patient satisfaction. ${ }^{19}$ As we experienced in our present cohort of patients, there has been a national trend of increased utilization of peripheral nerve blockade for postoperative pain manangement. ${ }^{20}$ This is consistent with the triple aim of health care of "improving the health (outcomes) of the (surgical) population".

While we did not undertake a formal cost-benefit analysis, it would intuitively appear more efficient and cost-effective to utilize a preoperative outpatient clinic venue rather than same-day surgery setting to educate patients about and to obtain informed consent for a regional analgesia block. A survey of US hospitals demonstrated that the average cost per minute of operating room time was $\$ 62 .{ }^{21}$ Our new regional analgesia process could thus result in $\$ 360$ (5 minutes) to $\$ 1080$ (15 minutes) cost savings per case. As facility with peripheral nerve blocks further improves, utilization of blocks may further increase, enhancing this cost-benefit. This is consistent with the triple aim of health care of "reducing per capita costs of care".

In addition to examining other ways to reduce surgical delays due to regional analgesia, efforts to further improve the individual patient experience with regional analgesic blockade represent potential research topics. These include 1) comparing anxiety and satisfaction in patients consented prior to vs on the day of surgery; 2) identifying and reducing learning and language barriers in the informed consent process; 3) optimizing preoperative patient education and health literacy; and 4) implementing culturally sensitive and language-specific, tablet-based decision support tools. These latter two topics would afford the opportunity to create and implement additional, more refined and focused preoperative multimedia patient education materials. Finally, given the repetitive and standardized nature of the present patient education and consent process, it could be incorporated and studied as an element of a surgical checklist.

A potential limitation of our study was misclassification bias of the cause of a surgical delay by the nonimpartial circulating surgical nurse; however, the cause of every so-noted delay was confirmed by the ostensibly impartial Director of Perioperative Services. A Hawthorne effect may have occurred if the preoperative providers changed their behavior, knowing their performance was being evaluated. However, these clinical providers were "not" informed of the additional purpose of the existing, longitudinal operational data collection.

Our study would have been strengthened if we had collected data on the throughput time for the entire patient encounter on the day of surgery by our RAPS. Unfortunately, given the existing, predetermined timestamps in our electronic medical record, this discrete time epoch could not be validly determined as an outcome variable. 


\section{Conclusion}

When performed before the day of surgery, an electronic surgical order, patient education, and informed consent for regional blockade for postoperative analgesia are associated with reduced block-related operating room delays. A preoperative management clinic can serve as a venue to achieve this objective, thereby adding value by decreasing delays on the day of surgery. This more judicious use of time, money, and personnel is another measurable benefit of a full-service preoperative management clinic. This approach also likely enhances the two important constructs of patient-centered care and shared decision making.

\section{Acknowledgments}

Attribution: Department of Anesthesiology and Perioperative Medicine, UAB, and UAB Hospital. The financial support was provided by the Department of Anesthesiology and Perioperative Medicine, UAB. This study was presented at the 40th Annual Regional Anesthesiology and Acute Pain Medicine Meeting, May 14-16, 2015, Las Vegas, NV, USA.

\section{Disclosure}

The authors report no conflicts of interest in this work.

\section{References}

1. Carli F, Kehlet H, Baldini G, et al. Evidence basis for regional anesthesia in multidisciplinary fast-track surgical care pathways. Reg Anesth Pain Med. 2011;36(1):63-72.

2. Tait AR, Teig MK, Voepel-Lewis T. Informed consent for anesthesia: a review of practice and strategies for optimizing the consent process. Can J Anaesth. 2014;61(9):832-842.

3. Jlala HA, French JL, Foxall GL, Hardman JG, Bedforth NM. Effect of preoperative multimedia information on perioperative anxiety in patients undergoing procedures under regional anaesthesia. Br JAnaesth. 2010; 104(3):369-374.

4. Gliner JA, Morgan GA, Leech NL. Randomized experimental and quasi-experimental designs. Research Methods in Applied Settings: An Integrated Approach to Design and Analysis. 2nd ed. New York: Routledge; 2009:55-72.
5. Berwick DM, Nolan TW, Whittington J. The triple aim: care, health, and cost. Health Aff (Millwood). 2008;27(3):759-769.

6. Stiefel M, Nolan K [homepage on the Internet]. A guide to measuring the Triple Aim: population health, experience of care, and per capita cost. IHI Innovation Series White Paper; 2012. Available from: www. IHI.org. Accessed October 30, 2013.

7. Cyriac J, Cannesson M, Kain Z. Pain management and the perioperative surgical home: getting the desired outcome right. Reg Anesth Pain Med. 2015;40(1):1-2.

8. Vetter TR, Jones KA. Perioperative surgical home: perspective II. Anesthesiol Clin. 2015;33(4):771-784.

9. Berwick DM. What 'patient-centered' should mean: confessions of an extremist. Health Aff (Millwood). 2009;28(4):w555-w565.

10. Kon AA. The shared decision-making continuum. JAMA. 2010; 304(8):903-904.

11. Vetter TR, Boudreaux AM, Jones KA, Hunter JM Jr, Pittet JF. The perioperative surgical home: how anesthesiology can collaboratively achieve and leverage the triple aim in health care. Anesth Analg. 2014;118(5): 1131-1136.

12. Goeddel LA, Porterfield JR Jr, Hall JD, Vetter TR. Ethical opportunities with the Perioperative Surgical Home: disruptive innovation, patientcentered care, shared decision making, health literacy, and futility of care. Anesth Analg. 2015;120(5):1158-1162.

13. Ankuda CK, Block SD, Cooper Z, et al. Measuring critical deficits in shared decision making before elective surgery. Patient Educ Couns. 2014;94(3):328-333.

14. Elwyn G, Frosch D, Thomson R, et al. Shared decision making: a model for clinical practice. J Gen Intern Med. 2012;27(10):1361-1367.

15. Neal JM, Barrington MJ, Brull R, et al. The second ASRA practice advisory on neurologic complications associated with regional anesthesia and pain medicine: executive summary 2015. Reg Anesth Pain Med. 2015;40(5):401-430.

16. Marhofer P, Harrop-Griffiths W, Kettner SC, Kirchmair L. Fifteen years of ultrasound guidance in regional anaesthesia: part 1. Br J Anaesth. 2010;104(5):538-546.

17. Marhofer P, Harrop-Griffiths W, Willschke H, Kirchmair L. Fifteen years of ultrasound guidance in regional anaesthesia: part 2-recent developments in block techniques. Br JAnaesth. 2010;104(6):673-683.

18. Neal JM. Ultrasound-guided regional anesthesia and patient safety: update of an evidence-based analysis. Reg Anesth Pain Med. 2016;41(2):195-204.

19. Kessler J, Marhofer P, Hopkins PM, Hollmann MW. Peripheral regional anaesthesia and outcome: lessons learned from the last 10 years. $\mathrm{Br} J$ Anaesth. 2015;114(5):728-745.

20. Chelly JE, Ghisi D, Fanelli A. Continuous peripheral nerve blocks in acute pain management. Br J Anaesth. 2010;105(suppl 1): i86-i96.

21. Macario A. What does one minute of operating room time cost? J Clin Anesth. 2010;22(4):233-236.
Local and Regional Anesthesia

Publish your work in this journal

Local and Regional Anesthesia is an international, peer-reviewed, open access journal publishing on the development, pharmacology, delivery and targeting and clinical use of local and regional anesthetics and analgesics. The journal is included in PubMed, and welcomes submitted papers covering original research, basic science, clinical studies,

\section{Dovepress}

reviews and evaluations, guidelines, expert opinion and commentary, case reports and extended reports. The manuscript management system is completely online and includes a very quick and fair peer-review system, which is all easy to use. Visit http://www.dovepress.com/ testimonials.php to read real quotes from published authors. 